\title{
A Walk through Johnny von Neumann's Garden
}

\author{
Freeman Dyson
}

\section{Foundations of Mathematics}

Johnny von Neumann left behind him six massive volumes of collected works, assembled and edited by Abraham Taub [1]. The collected works are his garden, containing a large and heterogeneous set of objects that he planted. Each of them grew from a seed, from an idea or a problem that came into his head. He developed the idea or solved the problem and then wrote it down and published it. He wrote fast and published fast, so that the flowers are still fresh. For my talk this morning I decided to take a walk through the garden and see what I could find. Luckily only two of the papers are in Hungarian. He wrote mostly in German until he came to live permanently in the United States at the age of thirty, and after that in English.

Johnny was educated at the famous Lutheran High School in Budapest from age ten to age eighteen. There he had excellent teachers and even more excellent schoolmates. One of the schoolmates was Eugene Wigner, who became an outstanding physicist and a lifelong friend. But Johnny's father understood that the Lutheran High School was not giving Johnny everything he needed. Johnny had a passion for mathematics going far beyond what the school could teach. So his father hired Michael Fekete, a mathematician from the University of Budapest, to work with Johnny at home. The first flower in Johnny's garden is a paper, "On the position of zeroes of certain minimum polynomials" [2], published jointly by Fekete and von Neumann when Johnny was

Freeman Dyson is professor emeritus at the Institute for Advanced Study, Princeton. His email address is dyson@ ias.edu.

Talk given at Brown University, Providence, Rhode Island, May 4, 2010.

DOI: http://dx.doi.org/10.1090/noti942 eighteen. The style of the paper is dry and professional, following the tradition set by Euclid two thousand years earlier. Almost everything that Johnny wrote as a mathematician is in the Euclidean style, stating and proving theorems one after another with no wasted words.

Although the subject of his first paper was probably suggested by Fekete, the style is already recognizable as Johnny's. Johnny's unique gift as a mathematician was to transform problems in all areas of mathematics into problems of logic. He was able to see intuitively the logical essence of problems and then to use the simple rules of logic to solve the problems. His first paper is a fine example of his style of thinking. A theorem which appears to belong to geometry, restricting the possible positions of points where some function of a complex variable is equal to zero, is transformed into a statement of pure logic. All the geometrical complications disappear and the proof of the theorem becomes short and easy. In the whole paper there are no calculations, only verbal definitions and logical deductions.

The next flower in the garden is Johnny's first solo paper, "On the introduction of transfinite numbers" [3], which he published at age nineteen. This shows where his strongest interests lay at the beginning of his career when he was a young bird ready to leave the nest and stretch out his mathematical wings. His dominating passion then and for the next five years was to understand and reconstruct the logical foundations of mathematics. He was lucky to arrive on the scene at the historical moment when confusion about the foundations of mathematics was at a maximum. In the nineteenth century, Georg Cantor had greatly enlarged the scope of mathematics by creating a marvelous theory of transfinite numbers, giving precise definitions to a vast hierarchy of infinities. Then, at the beginning of the twentieth century, 
Bertrand Russell and other critics discovered that Cantor's theory led to logical contradictions. Russell's paradox threw doubt not only onto Cantor's creation of a new world of infinities but also onto the established concepts of classical mathematics. Johnny became aware as soon as he began to talk with Fekete and to read the mathematical literature that mathematics was in a state of crisis. Since Cantor's mathematical reasoning had led to logical absurdities, nobody knew how to draw the line between reliable mathematics and imaginative nonsense. Johnny decided at the age of nineteen that it was his task to resolve the crisis and to put mathematics back onto a firm logical foundation.

The first paragraph of Johnny's first solo paper consists of a single sentence: "The purpose of this work is to make the idea of Cantor's ordinal numbers unambiguous and concrete." The rest of the paper provides a new definition of ordinal numbers and demonstrates that the new definition leads to the same results as Cantor's old definition. Johnny makes no claim to have resolved the crisis that arose from Cantor's theory. He has only made the crisis more acute by giving Cantor's concepts a sharper definition. To make the crisis more acute means to understand it better, and to understand it better is the first step toward resolving it.

Johnny's second solo paper, "An axiomatization of set theory" [4], appeared two years later when he was twenty-one years old and a student at the University of Berlin. Set theory means the theory of things and collections of things, considering only their logical relationships and forgetting about their individual qualities. From the point of view of set theory, you and I and stars and planets and words and numbers are all just things and are all treated the same way. Axiomatization means to describe set theory in the same style that Euclid used to describe geometry two thousand years ago, building the theory by logical deduction from a few basic assumptions which he called axioms. Johnny found a new set of axioms for set theory. He hoped that his new axioms could serve as a consistent logical basis for all the useful parts of mathematics while avoiding the paradoxes. But he was well aware that his consistent basis for mathematics was a hope and not a proven reality.

The essential novelty of Johnny's axioms was to introduce two species of objects, which he called "one-things" and "two-things". He used these abstract names in order to avoid possibly misleading impressions that might arise from using more familiar words. To make Johnny's ideas easier to understand, I will use the names "sets" for onethings and "classes" for two-things. So Johnny had a version of set theory with two kinds of objects: the sets, which are in some sense small enough to be handled collectively by the normal rules, and the classes, which are in some sense too big to be handled collectively. The axioms are constructed so that the "class of all sets" exists as a welldefined object. It is a class but not a set. Neither the "set of all sets" nor the "class of all classes" exists in the theory. This simple trick, using different names and different rules for small and large collectives, allows Johnny to avoid the logical paradoxes. The paradoxes arose in the older versions of set theory from using the concept "set of all sets" too freely. In Johnny's new version, this concept is forbidden, but the "class of all sets" is allowed, providing the framework for a logical construction of mathematics. The class of all sets is the universe of mathematics, the framework within which all mathematical collectives are defined.

Before writing his paper, Johnny had been talking with David Hilbert in Göttingen. Hilbert was forty years older than Johnny and was the most famous mathematician in the world. Hilbert was passionately promoting a program for resolving the crisis of mathematics by solving the Entscheidungsproblem, the decision problem. To solve the decision problem meant to find a formal method of deciding the truth or falsehood of every mathematical statement. If he could solve the decision problem, that would show that the axioms of mathematics were both consistent and categorical. To be consistent means that they can never prove both a statement and its negation. To be categorical means that for every statement the axioms prove either the statement or its negation. Hilbert proclaimed, with all his authority as spiritual father of mathematicians, that to resolve the crisis of mathematics it was necessary to find a set of axioms that were proven to be both consistent and categorical. Mathematics would only rest on a firm logical foundation if every meaningful mathematical statement could be proved true or false.

At the end of his axiomatization paper, Johnny puts a brief and modest summary of his claims. He does not claim to have resolved the crisis of mathematics. He claims only to have opened the way to a possible resolution by finding a set of axioms that is not known to be self-contradictory. He has not proved that his axioms are consistent, and he has not proved that they are categorical. He ends his paper with two sentences expressing not very diplomatically his skepticism about Hilbert's program: "Even Hilbert's approaches are here powerless, for this objection concerns the categoricity and not the consistency of set-theory. All that we can do now is to recognize that another argument against set-theory has arisen, and that we see no way ahead leading to rehabilitation."

Three years later Johnny published two much longer papers about the foundations of mathematics. One was "On Hilbert's proof theory" [5]. The other was his Ph.D. thesis, with the title "The axiomatization of set theory" [6], an expanded version of the 1925 paper. These two papers show that Johnny was still desperately trying to rescue 
mathematics by following Hilbert's program. Johnny was stuck. He had created a simple and beautiful new set of axioms, which were later shown by Kurt Gödel to be exactly what was needed for understanding the true nature of mathematics, but he did not know what to do with them. At that point, he gave up trying to rescue mathematics and devoted the rest of his life to other things.

Another three years later, in 1931, Kurt Gödel in Vienna proved two theorems that totally devastated the Hilbert program. Gödel proved that no system of axioms for mathematics could be categorical and that no system of axioms could prove itself to be consistent. After Gödel, mathematics could never be the unique compendium of absolute truth that mathematicians from Euclid to Hilbert had imagined. After Gödel, mathematics was a free creation of the human mind, with truth and falsehood depending on human tastes and preferences. For Hilbert and many of his contemporaries, the discoveries of Gödel appeared to be a disaster. Their hopes of building a unique and solid foundation for mathematics had collapsed. But Johnny understood immediately that the new freedom created by Gödel was a gain and not a loss. Johnny said in a public lecture that Gödel was the greatest logician since Aristotle. Johnny regretted that he had not made Gödel's discoveries himself three years earlier, but he was happy to see that Gödel used his 1925 system of axioms with separate names for sets and classes. Johnny was proud to have made a substantial contribution to the foundations of the new mathematics.

\section{Games and Quanta}

The next flower, "Theory of party games" [7], comes from a different corner of the garden. At the age of twenty-four, Johnny had become a professional mathematician with a position as instructor at the University of Berlin. He enjoyed the night life of Berlin and was intrigued by the logic of games such as poker and baccarat in which the outcome depends on a mixture of luck and skill. The question, whether a logical strategy exists for a player to have the best chance to win such games, had been raised by the French mathematician Émile Borel. Borel had asked the question but was unable to answer it. Johnny found the answer, which turned out to be a deep mathematical theorem. For a game with only two players, there exists a unique strategy which gives each of them the best outcome on the average. The proof that such a strategy exists is another fine example of Johnny's style, reducing a problem of calculation to a problem of logic.

The optimum strategy usually requires a large element of randomness so that the moves of the players are truly unpredictable. Player A must throw dice to decide how to move so that Player
B cannot win by predicting what Player A will do. In the game of poker the throw of the dice will occasionally require Player A to bet high on a weak hand, a move that is called bluffing. If Player A never bluffs, Player B can win by guessing more accurately the strength of Player A's cards. At the end of his paper Johnny writes, "The agreement of the mathematical results with the empirically known rules of successful gambling, for example the necessity of bluffing in poker, can be considered as experimental confirmation of our theory."

For games with three or more players, Johnny found no such elegant solution to the problem. To have the best chance of winning a game with three players, Player A must bribe or threaten Player B to form a coalition against Player $C$. The players must compete for the roles of the winners, A and B, and try to escape the role of the loser, C. The result of the competition is decided by personal willpower or spite and not by mathematics. At the end of his discussion of the three-person game, Johnny says, "The decisive factor, which is altogether absent from the orderly and equitable two-person game, is combat."

In another corner of the garden there is a little flower all by itself, a short paper with the title "The division of an interval into a denumerable infinity of identical parts" [8]. This solves a problem raised by the Polish mathematician Hugo Steinhaus. I met Steinhaus after World War II in America. He was one of the few survivors of the group of brilliant mathematicians who emerged in Poland between the wars. Half of them were Jewish and half were Gentiles. The chance of survival was about the same for both, since those who emigrated were mostly Jews and those who survived in Poland were all Gentiles. Johnny solved the Steinhaus problem quickly and never returned to it. The theorem that he proved is counterintuitive, and the proof is astonishing. The theorem is about sets of points on an interval. An interval means a finite piece of a straight line. A denumerable infinity means a collection of objects that can be labeled with whole numbers, $1,2,3, \ldots$, all the way to infinity. The theorem says that there exists a collection of sets of points $S 1, S 2, S 3, \ldots$, with the following properties: (1) Every point on the interval belongs to exactly one Sj. (2) The sets Sj are identical in all respects except for position, each $\mathrm{Sj}$ being obtained from any other by displacing it bodily through a certain distance along the line.

The theorem is counterintuitive because it is impossible to visualize the sets Sj. If you try to imagine how the points of the set $\mathrm{Sj}$ are arranged near the ends, you fail. You fail because the sets are nonmeasurable, and nobody has ever visualized a nonmeasurable set of points. Nonmeasurable sets cannot be constructed using any of the familiar tools of geometry. Johnny's proof of the theorem is astonishing because it is totally abstract. He never 
even mentions the geometry of the sets Sj. He gives no clue to their appearance or their construction. He proves their existence by reducing it to a proposition in pure logic and proves the proposition by purely logical arguments. This little paper is the most extreme manifestation of the Johnny style.

During his Berlin years, Johnny made frequent visits to Göttingen, where Heisenberg had recently invented quantum mechanics and Hilbert was the presiding mathematician. Hilbert was intensely interested in quantum mechanics and encouraged collaboration between mathematicians and physicists. From the point of view of Hilbert, quantum mechanics was a mess. Heisenberg had no use for rigorous mathematics and no wish to learn it. Dirac made free use of his famous deltafunction, which was defined by a mathematical absurdity: being infinite at a single point and zero everywhere else. When Hilbert remarked to Dirac that the delta-function could lead to mathematical contradictions, Dirac replied, "Did I get into mathematical contradictions?" Dirac knew that his delta-function was a good tool for calculating quantum processes, and that was all he needed. Twenty years later, Laurent Schwartz provided a rigorous basis for the delta-function and proved that Dirac was right. Meanwhile, Johnny worked with Hilbert and published a series of papers cleaning up the mess. For several years, quantum mechanics was Johnny's main interest. In 1932 he published the book Mathematical Foundations of Quantum Mechanics [9], which occupies a substantial piece of his garden.

Johnny's book was the first exposition of quantum mechanics that made the theory mathematically respectable. The concepts were rigorously defined and the consequences rigorously deduced. Much of the work was original, especially the chapters on quantum statistics and the theory of measurement. I read the book in 1946 when I was still a pure mathematician but already intending to switch my attention to physics. I found it enormously helpful. It gave me what I needed, a mathematically precise statement of the theory, explaining the fine points that the physicists had been too sloppy to mention. From that book I learned most of what I know about quantum mechanics. But then, after I had made the transition to physics and had begun to read the current physics journals, I was surprised to discover that nobody in the physics journals ever referred to Johnny's book. So far as the physicists were concerned, Johnny did not exist. Of course, their ignorance of Johnny's work was partly a problem of language. The book was in German, and the first English translation was only published in 1955. But I think even if the book had been available in English, the physicists of the 1940s would not have found it interesting. That was the time when the culture of physics and the culture of mathematics were most widely separated. The culture of physics was dominated by people like Oppenheimer who made friends with poets and art historians but not with pure mathematicians. The culture of mathematics was dominated by the Bourbaki cabal, which tried to expunge from mathematics everything that was not purely abstract. The gap between physics and mathematics was as wide as the gap between science and the humanities described by C. P. Snow in his famous lecture on the two cultures. Johnny was one of the very few people who were at home in all four cultures: in physics and mathematics, and also in science and the humanities.

The central concept in Johnny's version of quantum mechanics is the abstract Hilbert space. Hilbert space is the infinite-dimensional space in which quantum states are vectors and observable quantities are linear operators. Hilbert had defined and explored Hilbert space long before quantum mechanics made it useful. The unexpected usefulness of Hilbert space arises from the fact that the equations of quantum mechanics are exactly linear. The operators form a linear algebra, and the states can be arranged in multiplets defined by linear representations of the algebra. Johnny liked to formulate physical problems in abstract and general language, so he formulated quantum mechanics as a theory of rings of linear operators in Hilbert space. A ring means a set of operators that can be added or subtracted or multiplied together but not divided. Any physical system obeying the rules of quantum mechanics can be described by a ring of operators. Johnny began studying rings of operators to find out how many different types of quantum systems could exist.

After Johnny had published his quantum mechanics book, he continued for several years to develop the theory of rings of operators. The third volume of his collected works consists entirely of papers on rings of operators. He published seven long papers with a total of more than five hundred pages. I will not discuss these monumental papers this morning. They contain Johnny's deepest work as a pure mathematician. He proved that every ring of operators is a direct product of irreducible rings that he called factors. He discovered that there are five types of factor, of which only two were previously known. Each of the types has unique and unexpected properties. Exploring the ocean of rings of operators, he found new continents that he had no time to survey in detail. He left the study of the three new types of factor unfinished. He intended one day to publish a grand synthesis of his work on rings of operators. The grand synthesis remains an unwritten masterpiece, like the eighth symphony of Sibelius.

The quantum mechanics book is the last item on my list of flowers that Johnny published in German. It was published in 1932 when he was dividing his time equally between Berlin and Princeton. In 
the same year he began writing papers in English. One of his first papers to appear in English was "Proof of the quasi-ergodic hypothesis" [10], which he published in the Proceedings of the National Academy of Sciences to make sure that American mathematicians would read it. This paper solved an important problem in classical mechanics using the same concept of Hilbert space that he had used to solve problems in quantum mechanics. A classical dynamical system is said to be ergodic if after we put it into an initial state and then leave it alone for an infinite time, it comes arbitrarily close to any final state with probability independent of the initial state. Johnny proved that under certain clearly specified conditions, a system is ergodic if and only if there exist no constants of the motion. A constant of the motion means a quantity depending on the state of the system which does not change as the system moves forward in time. Johnny's theorem provides a firm mathematical basis for the assumptions that are customarily made by physicists using classical statistical mechanics. Translated into the sloppy language used by physicists, the theorem says that the timeaverage of any single trajectory of the system over a long time is equal to the statistical average of all trajectories. Even more sloppily, physicists say that time-averages are equal to ensemble averages, and we use the word ensemble to mean the set of all states of the system.

One of the American mathematicians who read Johnny's paper in the Proceedings of the National Academy was Garrett Birkhoff. Garrett was the son of George Birkhoff, and both father and son were famous mathematicians. Garrett and Johnny became close friends, and Garrett came to Princeton for frequent visits. After Johnny died, Garrett wrote a memoir about the work that Johnny did in the 1930s. Here is a sentence from Garrett's memoir: "Anyone wishing to get an unforgettable impression of the razor edge of von Neumann's mind need merely try to pursue this chain of exact reasoning for himself, realizing that often five pages of it were written down before breakfast, seated at a living room writing-table in a bathrobe."

A minor offshoot of Johnny's thinking about operators in Hilbert space was his invention of continuous geometry, a new kind of geometry in which the dimension of a subspace is a continuous variable. A couple of short papers, "Continuous geometry" [11] and "Examples of continuous geometries" [12], are to be found in his garden. These papers were published in 1936 when Johnny was settled in Princeton. Johnny writes at the beginning, "We will give only the axioms, some commentaries on them, and then the main definitions and results. A detailed account will appear soon in a mathematical periodical." This is a promise that was never fulfilled. From this time in his life onward he made many such broken promises. He got into the habit of working on a problem, solving it to his own satisfaction, and then not taking the time to publish the results in detail. He gave lectures in Princeton on continuous geometry. His lecture notes were published in a book Continuous Geometry, which appeared in 1960 after his death. The book is boring. It is probably the most boring stuff that ever appeared under Johnny's name. You can tell from the book that Johnny was already bored by continuous geometry while he was giving the lectures. He had good reasons for not publishing the notes while he was alive. He had no need to publish or perish. He was a tenured professor at the Institute for Advanced Study. After 1936 he published only stuff that he considered important and not boring. He became increasingly interested in a wide range of subjects outside pure mathematics. He had, after all, earned a degree in chemical engineering at the ETH in Zurich at the same time as he was studying mathematics in Budapest.

\section{Bombs and Computers}

The next flower is a report, Theory of Detonation Waves [13], written in 1942, presenting a scholarly and thorough analysis of what happens when chemical high explosives detonate. Johnny had seen his homeland, Hungary, dismembered as a result of military defeat in 1918 . He was even more eager than other European Jews to join the fight against Hitler. He was delighted to apply his mathematical skills and his knowledge of engineering to military problems and became a consultant to the United States Army before the United States went to war in 1941 . His 1942 report was one of a series providing a theoretical basis for the improvement of military explosives. Military explosives are a delicate compromise between two conflicting requirements: they should detonate with maximum efficiency when fired in anger and should resist detonation with maximum safety when exposed to gunfire or accidental explosions nearby. When you are trying to find the best compromise, it is a great help to have a consultant who understands the chemistry as well as the mathematics.

Johnny's report does not discuss particular weapons but supplies a mathematical theory that designers of weapons can use to optimize designs. When he began work for the military, the applications were to artillery shells and antisubmarine depth charges. In 1943 he was invited by his friend Robert Oppenheimer to visit Los Alamos and apply his ideas to the design of nuclear weapons. His understanding of shock waves made a big contribution to the success of the Los Alamos project. At Los Alamos he saw monstrous numerical calculations carried out laboriously by gangs of human computers. He began to think seriously about the possibility of electronic computers that could do such calculations better and faster than humans. In 1944 he met Herman Goldstine, who was then a 
young army officer involved in a project to build a real electronic computer, the ENIAC, at the University of Pennsylvania. Johnny and Herman became close friends. Herman later said of Johnny, "While he was indeed a demi-god, he had made a detailed study of humans and could imitate them perfectly. Actually he had great social presence, a very warm, human personality, and a wonderful sense of humor." They worked out a plan to do something spectacular with computers as soon as the war was over. In Johnny's garden there is a paper, "On the principles of large scale computing machines" [14], describing their plans. I won't say more about this, since Johnny's work on computers is covered by other speakers.

I got to know Johnny personally when I came to the Institute for Advanced Study in 1948. He was then actively engaged in building the institute computer and learning how to use it. He understood from the beginning that two of the most important uses of the machine would be to predict weather and to model climate. He hired engineers to build the machine and meteorologists to use it. The chief engineer was Julian Bigelow, and the chief meteorologist was Jules Charney. Each of them had a gang of young people to do the heavy work, persuading a totally new kind of machine to produce some real science. I enjoyed very much the young people, with their rowdy conversation and irreverent behavior. There was an amusing clash of cultures between these young hooligans and the older members of the institute. As Einstein wrote to his friend the queen of the Belgians when he arrived at the institute in 1933, Princeton was a quaint and ceremonious village populated by demigods on stilts. The culture of the older members was based on formal politeness and respect for the academic hierarchy. Johnny and I were on the side of the hooligans.

When Johnny died, the institute quickly got rid of the computer project, and the older culture reasserted itself. No more hooligans were hired, and the breath of fresh air that they had brought to the institute was blown away with them to UCLA and MIT. In 1980 the institute celebrated its fiftieth birthday by publishing a volume with the title $A$ Community of Scholars, 1930-1980, consisting of biographies and bibliographies of the members. Not one of the young hooligans who built the machine and predicted the weather is mentioned in the book. They were not scholarly enough to be officially recognized as belonging to the institute. But there is a flower in Johnny's garden, a paper, "Numerical integration of the barotropic vorticity equation" [15], by Charney, Fjortoft, and von Neumann, describing their first attempts to predict weather. Since the institute computer was not yet running, they did their calculations with the ENIAC. Using the ENIAC, the numerical simulation moved ahead in time more slowly than the weather that it was supposed to simulate, so there was no real prediction. At the end they express the hope that the institute computer will be fast enough to keep ahead of real time. Four years later, when Johnny's machine and others like it were running, their hopes were fulfilled. Johnny then announced that a prediction of weather twenty-four hours ahead could be done in less than an hour. That was as far as he was able to go toward his dream of understanding climate. One year later, he was diagnosed with terminal cancer, and three years later he was dead.

\section{Summing Up}

In the last decade of his life, Johnny did not find time to write formal mathematical papers. Instead he wrote informal essays, sometimes addressed to his colleagues in the government agencies that supported his work and sometimes to the general public. The last two flowers in my tour of his garden are addressed to the public. They are thoughtful and beautifully written. He took a lot of trouble to think clearly and write simply. The first of the two was titled "The mathematician" [16]. It was published in 1947 as a chapter in a book of essays, The Works of the Mind, by a variety of authors. It is a swan song, summarizing in simple words the conclusions that Johnny had reached at the end of his life as a pure mathematician. He had devoted the best years of his life to pure mathematics, when he was, as Newton said of his own early years, "in the prime of my life for invention." From age nineteen to age twenty-seven he had struggled to build firm logical foundations for pure mathematics, preparing the ground for Gödel's discovery that no set of foundations could be complete. After the Gödel revolution, he took advantage of the new freedom to experiment with logical foundations for quantum mechanics and for the discipline that was later given the name of computer science. His essay "The mathematician" describes the development of mathematics as a free creation of the human mind, with foundations either borrowed from empirical science or freely invented.

The main message of Johnny's essay is stated at the end in words that have become famous among mathematicians: "As a mathematical discipline travels far from its empirical source, or still more, if it is a second and third generation only indirectly inspired by ideas coming from reality, it is beset with very grave dangers.... At a great distance from its empirical source, or after much abstract inbreeding, a mathematical subject is in danger of degeneration. At the inception the style is usually classical. When it shows signs of becoming baroque, then the danger signal is up. It would be easy to give examples, to trace specific evolutions into the baroque and the very high baroque, but this, again, would be too technical. In any event, 
whenever this stage is reached, the only remedy seems to me to be the rejuvenating return to the source, the reinjection of more or less directly empirical ideas. I am convinced that this was a necessary condition to conserve the freshness and the vitality of the subject, and that this will remain equally true in the future." Johnny probably had in mind his elaborate fiddling with continuous geometry as an example of very high baroque, and his plunge into the empirical world of computer science as the rejuvenating return to the source.

After this farewell to pure mathematics, the last seven years of Johnny's life were divided between running the computer project in Princeton and advising the government in Washington. During this period he became known to the public as a military hardliner. For a few years he publicly advocated a preventive war against the Soviet Union. He became deeply involved with high-level committees considering problems of military strategy. One of the committees, known to historians as the von Neumann Committee, advocated the fateful decision to base United States strategy on a force of intercontinental ballistic missiles, combining the technologies of multistage rocketry and hydrogen bombs. This decision would make it technically possible for the United States to destroy the Soviet Union in forty minutes, with the inevitable consequence that the Soviet Union would be able to destroy the United States with a similar force of missiles a few years later.

The idea of a preventive nuclear war conveys today an impression of militarism gone mad. But to the generation that lived and suffered through the 1930s, the idea had another meaning. It was widely held, especially by liberal intellectuals, that the French and British governments had behaved in a cowardly and immoral fashion when they failed to march into Germany in 1936 to stop Hitler from remilitarizing the Rhineland. A preventive war in 1936, when Germany was still effectively disarmed and incapable of serious resistance against invading forces, might have overturned Hitler's regime in a few days and saved the fifty million human beings who were to die in World War II. We cannot know whether a preventive war in 1936 would have been either feasible or effective. We know only that the idea of preventive war as a morally acceptable option was widely accepted by the people of Johnny's generation, who looked back to 1936 as a tragically missed opportunity. To them, the idea of forestalling a terrible catastrophe by a bold preventive action was neither insane nor criminal.

Johnny argued in the 1940s that America was facing the same choice that France and Britain faced in 1936. The Soviet Union was then just beginning to build the industrial base for the mass production of nuclear weapons. Johnny saw the 1940s as the last chance for America to overthrow the Stalin regime, as 1936 had been the last chance to overthrow Hitler, without a war of annihilation. He saw a preventive war in the 1940s as preferable, not only for America but for humanity as a whole, to a war of annihilation later. I am not saying that he was right. I consider it unlikely that preventive war could have achieved its objective either in 1936 or in the 1940s. I am only saying that to talk of Johnny's advocacy of preventive war without mentioning the events of 1936 which dominated his perception of the moral issues is to miss the main point of his argument.

The last flower on my tour of Johnny's garden is a paper written for the general public and published in Fortune magazine in June 1955, two months before the onset of his fatal illness. The title is "Can we survive technology?" [17]. Johnny is now no longer concerned with the intellectual problems of mathematicians but with the human problems of war and peace, nuclear weapons and nuclear power, global warming and climate control, computers changing the rules of economics and politics. In the last seven years of his life, as he moved into the centers of power in Washington and made friends with generals and politicians, he understood that the urgent problems of society were human rather than technical. His view of human nature was bleak. "It is just as foolish to complain that people are selfish and treacherous as it is to complain that the magnetic field does not increase unless the electric field has a curl. Both are laws of nature." His view of the future was equally bleak. "Present awful possibilities of nuclear warfare may give way to others even more awful. After global climate control becomes possible, perhaps all our present involvements will seem simple. We should not deceive ourselves. Once such possibilities become actual, they will be exploited.... The one solid fact is that the difficulties are due to an evolution that, while useful and constructive, is also dangerous. Can we produce the required adjustments with the necessary speed? The most hopeful answer is that the human species has been subjected to similar tests before and seems to have a congenital ability to come through, after varying amounts of trouble. To ask in advance for a complete recipe would be unreasonable. We can specify only the human qualities required: patience, flexibility, intelligence." Johnny possessed these qualities himself. They are still the qualities that we need in order to have the best chance of survival as we move into the world that he created.

\section{References}

[1] John von Neumann, Collected Works, A. H. Taub, ed., Pergamon Press, New York, 1961-1963.

[2] __ Über die Lage der Nullstellen gewisser Minimalpolynomen (with M. Fekete), Jahresbericht 31, 125-138 (1922).

[3] __ Zur Einführung der transfiniten Zahlen, Acta Szeged. 1, 199-208 (1923). 
[4] _ E Eine Axiomatisierung der Mengenlehre, J. für. Math 154, 219-240 (1925).

[5] __ Zur Hilbertschen Beweistheorie, Math. Zeitschr. 26, 1-46 (1927).

[6] _ Die Axiomatisierung der Mengenlehre, Math. Zeitschr. 27, 669-752 (1928).

[7] __ Zur Theorie der Gesellschaftsspiele, Math. Ann. 100, 295-320 (1928).

[8] __ Die Zerlegung eines Intervalles in abzählbar viele kongruente Teilmengen, Fund. Math. 11, 230-238 (1928).

[9] __ Mathematische Grundlagen der Quantenmechanik, Springer, Berlin, 1932.

[10] __ Proof of the quasi-ergodic hypothesis, Proc. Nat. Acad. Sci. 18, 70-82 (1932).

[11] __ Continuous geometry, Proc. Nat. Acad. Sci., U.S.A. 22, 92-100 (1936).

[12] __, Examples of continuous geometries, Proc. Nat. Acad. Sci. U.S.A. 22, 101-108 (1936).

[13] __ , Theory of Detonation Waves, A progress report to April 1, 1942, Office of Scientific Research and Development Section B-1, report 549, 34 pp. (1942).

$[14] \ldots$ _ On the principles of large-scale computing machines (with H. H. Goldstine), Office of Research and Inventions, Navy Department, unpublished (1946).

[15] __ Numerical integration of the barotropic vorticity equation (with J. G. Charney and R. Fjortoft), Tellus 2, 237-254 (1950).

[16] _ _ The mathematician. In The Works of the Mind, R. B. Heywood, ed., University of Chicago Press, 180-196, 1947.

[17] __, Can we survive technology? Fortune (June 1955).

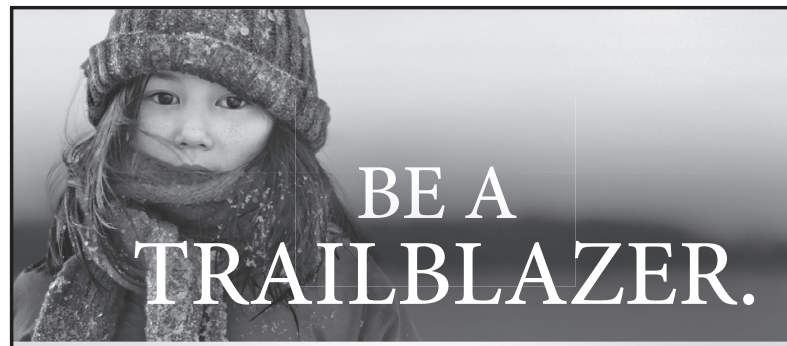

The University of Manitoba is a place where you can define you future and your career. Our commitment to discovery and community encompasses everything we do, from the education we offer students to the workplace we create for staff.

The University of Manitoba offers excellent benefits, world-class facilities and an inspirational environment where you can shape your career and make a positive impact every day.

\section{Department Head \\ Department of Mathematics Faculty of Science}

The Faculty of Science at the University of Manitoba invites applications or nominations for the position of Head of the Department of Mathematics. The successful candidate will be appointed to the rank of Associate Professor or Professor, commensurate with qualifications and experience. The position as Head is normally for a five year period and will begin on July 1, 2013 or on a mutually agreed upon date. The successful candidate must have a Ph.D. in mathematics, and an outstanding record of accomplishment in both research and teaching. Strong academic leadership experience, including involvement in the creation of undergraduate and graduate programs, and excellent communication, interpersonal and organizational skills, are preferred. Position Number: AM993

The Department of Mathematics is in the midst of an extensive renewal program, and the department is seeking new leadership to guide it through these exciting developments. Existing vacancies and anticipated retirements over the next five years will give the new Head a unique opportunity to lead in the reshaping and renewal of both the research and teaching programs of the department. Applications should include a curriculum vitae and a brief statement indicating the applicant's vision of the future of research and teaching in mathematics. Applicants should also arrange to have three letters of reference sent directly to the address below. Review of applications will begin March 1, 2013 and will continue until the position is filled. Additional information about the department, and the position profile, can be found at www.math.umanitoba.ca.

Applications or nominations should be forwarded to Dr. Roy M. Roshko, Associate Dean, Faculty of Science, University of Manitoba, 247 Machray Hall, Winnipeg, Manitoba, Canada R3T 2N2, email: roy.roshko@ad.umanitoba.ca.

The University of Manitoba encourages applications from qualified women and men, including members of visible minorities, Aboriginal peoples and persons with disabilities. All qualified candidates are encouraged to apply, however, Canadians and permanent residents will be given priority. Application materials, including letters of reference, will be handled in accordance with the protection of privacy provisions of "The Freedom of Information and Protection of Privacy Act" (Manitoba). Please note that CVs may be provided to participating members of the search process.

Winnipeg has a great deal to offer, both culturally and recreationally, with a number of arts groups including the Royal Winnipeg Ballet, professional sports teams, outstanding restaurants, and opportunities for all types of outdoor activities in all seasons. The Winnipeg housing market is one of the most favorable in Canada. Further information can be found at www.tourismwinnipeg.com.

For more information on this and other opportunities, please visit: umanitoba.ca/employment
UNIVERSITY of MANitoba 